\title{
Stoichiometric Magnetite Grown by Infrared Nanosecond Pulsed Laser Deposition
}

Mikel Sanz ${ }^{1 *}$, Mohamed Oujja ${ }^{1}$, Esther Rebollar ${ }^{1}$, José F. Marco ${ }^{1}$, Juan de la Figuera ${ }^{1}$, Matteo

Monti $^{1}$, Alberto Bollero ${ }^{2}$, Julio Camarero ${ }^{2,3}$, Francisco J. Pedrosa ${ }^{2}$, Mar García-Hernández ${ }^{4}$ and Marta Castillejo ${ }^{1}$

${ }^{1}$ Instituto de Química Física Rocasolano, CSIC, 28006 Madrid, Spain

${ }^{2}$ IMDEA Nanoscience, Instituto Madrileño de Estudios Avanzados en Nanociencia, Campus Universidad Autónoma de Madrid, 28049 Madrid, Spain

${ }^{3}$ Departamento de Física de la Materia Condensada, Instituto Nicolás Cabrera, Campus Universidad Autónoma de Madrid, 28049 Madrid, Spain

${ }^{4}$ Instituto de Ciencias Materiales de Madrid, CSIC, 28049, Madrid, Spain

*Email: mikel.sanz@iqfr.csic.es

Tel.: +34915619400961023

Fax: +34915642461

\begin{abstract}
Pulsed laser deposition (PLD) is a versatile technique for the fabrication of nanostructures due to the possibilities it offers to control size and shape of nanostructured deposits by varying the laser
\end{abstract}


parameters. Magnetite nanostructures are currently promising materials to be used in computing, electronic devices and spintronic applications. For all these uses the fabrication of uniform nanostructured pure magnetite thin films is highly advantageous. In PLD of magnetite the laser irradiation wavelength and substrate temperature crucially affect the composition, crystallinity, surface structure and the magnetic properties of the grown samples. This work shows that the use of nanosecond IR laser at $1064 \mathrm{~nm}$ enhances the quality of the resulting magnetite thin films, compared to the extensively used UV wavelengths. Deposition at $1064 \mathrm{~nm}$ upon heating the substrate at $750 \mathrm{~K}$ produces thin films constituted by stoichiometric magnetite nanoparticles with sharp edges and sizes ranging from 80 to $150 \mathrm{~nm}$, with a Verwey transition at $119 \mathrm{~K}$ and a coercivity of 232 Oe at room temperature, close to those of pure bulk magnetite. Thus, IR PLD together with the use of self-prepared hematite sintered targets allows preparing low-cost pure magnetite nanostructured thin films.

\section{Keywords}

Pulsed laser deposition, Nanocrystalline Magnetite fabrication, Magnetic properties 


\section{Introduction}

Pulsed laser deposition (PLD) has been widespread used for the fabrication of metal, oxides and semiconductor nanostructured thin films due to the possibilities it offers to control the size, shape and crystalline phase of the nano deposits by varying the laser and other growth parameters.[1-3] In particular, the wavelength effect on the formation of nanostructured films produced by PLD has been studied in a very diverse range of materials ${ }_{[1-7]}$ and an increase of the nanoparticle mean diameter with wavelength has been reported. An important drawback of the laser deposition method is, however, the presence in the film of micrometer sized particulates. These particulates are generally attributed to ablation of loosely connected target flakes, splashing of molten target material and deep target heating for irradiation with a poorly absorbed wavelength that lead to explosion of bubbles and condensation in the high density plume.[4] Typically it has been shown that the use of UV laser wavelengths in PLD yields smoother films, with fewer and smaller particulates than those obtained when visible or IR lasers are used.[5-7]

Half-metallic ferromagnetic materials, characterized by $100 \%$ spin polarization and having only one spin-subband at the Fermi level, are being actively investigated.[8] One example is magnetite $\left(\mathrm{Fe}_{3} \mathrm{O}_{4}\right)$, the oldest known magnetic material that has attracted enormous attention due to its high Curie temperature $(858 \mathrm{~K}),[9]$ and a metal-insulator transition (Verwey transition) at 120 K.[10-12] Magnetite nanostructures are thus promising materials for computing and electronic devices, spintronic applications and biomedicine.[13-19] Their performance will be dependent on the chemical and physical characteristics of the nanoparticles and their surfaces. Thus, the control of the particle size, shape, distribution and crystallinity is of crucial importance for the development of applications. The fabrication method should allow for tailoring the size and surface chemistry of the magnetic nanoparticles to meet the specific application demands. 
Recent PLD investigations of magnetite thin films are routinely performed with UV lasers, generally in an attempt to minimize the presence of particulates on the resulting films, therefore disregarding the use of IR irradiation and its optimization for the production of quality magnetite films. Magnetite thin films have been grown by PLD using magnetite as target material on different substrates.[20-28] Other works have shown the possibility of low-cost fabrication of magnetite thin films by PLD using hematite $\left(\alpha-\mathrm{Fe}_{2} \mathrm{O}_{3}\right)$ targets.[29-40] The influence of the nature $[24,25,34,36]$ and temperature [26, 39] of the substrates, ambient pressure, [27, 28] or film thickness[31, 37] on the magnetic properties of the deposits has been studied.

In this work, we report on the growth of magnetite nanostructured films by PLD using nanosecond pulses at wavelengths of 213, 532, and $1064 \mathrm{~nm}$ on $\mathrm{Si}$ (100) substrates from self-prepared sintered hematite targets. The deposits were characterized by atomic force microscopy (AFM) to determine the surface morphology, by X-ray diffraction (XRD) to examine their crystallinity, and by micro-Raman and Mössbauer spectroscopies to determine their composition and stoichiometry. Magnetic characterization was carried out by superconducting quantum interference device magnetometry (SQUID) and magneto-optical Kerr effect (MOKE). The results extend previous knowledge on the modulation of the size, shape and polycrystallinity of the nanostructured films that can be achieved under different experimental conditions, namely laser wavelength and substrate temperature. The use of IR PLD as an alternative to more conventional UV PLD has shown to enhance the quality of the prepared magnetite thin films. This, in combination with the use of self-prepared hematite sintered targets, yields a low-cost procedure to prepare pure magnetite nanostructured thin films for different applications. 


\section{Experimental section}

The experimental PLD system consists of a stainless-steel vacuum deposition chamber pumped down to $2 \times 10^{-4} \mathrm{~Pa}$ by a turbo-molecular pump.[41] Hematite targets were prepared from a $>99 \%$ iron (III) oxide powder (Sigma Aldrich, particle size $<5 \mu \mathrm{m}$ ) that was pelletized into disks of $10 \mathrm{~mm}$ diameter and about $2 \mathrm{~mm}$ thickness using a hydrostatic press at $8 \mathrm{Ton}^{-2}$ followed by sintering at $1173 \mathrm{~K}$ in air for 8 hours. Targets were placed on a rotating sample holder and ablated with a Q-switched Nd:YAG laser (FWHM $15 \mathrm{~ns}, 10 \mathrm{~Hz}$ ) at the excitation wavelengths of 213,532 , and $1064 \mathrm{~nm}$. The laser beam was focused onto the target at $45^{\circ}$ to a spot area of $0.14 \mathrm{~mm}^{2}$ with a typical fluence (laser energy per unit area) of 8 times the ablation threshold fluence for each irradiation wavelength. The corresponding ablation threshold fluences were determined by measuring the minimum single pulse energy necessary to yield a luminous plume as detected by eye and the diameter of the irradiated region by the print left on an unplasticized polyvinyl chloride sheet. Based on the obtained ablation thresholds the used irradiation fluences were $0.8,2.4$ and $4 \mathrm{~J} \mathrm{~cm}^{-2}$ for 213, 532 and $1064 \mathrm{~nm}$, respectively. The Si (100) substrates were ultrasonically degreased in acetone and methanol for 10 minutes and mounted on a heating element that allows operation in a temperature range between 300 and $773 \mathrm{~K}$, as measured by a thermocouple. The substrates were placed in front of the target at a distance of $40 \mathrm{~mm}$. Deposits were grown by delivering 144000 pulses to the target (resulting in a deposition time of around 4 hours).

The morphology of the deposits was examined by AFM (Multimode 8, Bruker) in tapping mode, and the images were analyzed with the Nanoscope Analysis 1.40 software. The mean roughness $\left(\mathrm{R}_{\mathrm{a}}\right)$, determined from AFM analysis, is the arithmetic average of the deviations in height from the center plane of the sample and corresponds to the average of 5 independent 
measurements in different locations of the sample. The thickness values result also from the average of 5 different measurements in different positions. The size distribution and average diameter of the deposited nanoparticles were obtained by determining the nanoparticle diameters from the profiles of the height in the AFM images. For each image, 10 parallel profiles were acquired at ca. $200 \mathrm{~nm}$ intervals and the results of three different images were readily averaged. The crystalline quality and composition of the deposits were studied by XRD (Philips XPert) using $\mathrm{Cu} \mathrm{K}_{\alpha}(0.154184 \mathrm{~nm})$ radiation in the $\theta / 2 \theta$ configuration. Micro-Raman spectra were obtained with a confocal Renishaw Raman microscope, RM-2000, equipped with a Leica microscope, and an electrically refrigerated CCD camera. A Helium-Neon laser at $632.8 \mathrm{~nm}$ was used as excitation source (20 $\mathrm{mW}$ laser, 50x objective). Ten scans were collected and summed up over the $100-2000 \mathrm{~cm}^{-1}$ Raman shift range to provide good signal-noise ratios. The wavenumber resolution and acquisition time were $5 \mathrm{~cm}^{-1}$ and $10 \mathrm{~s}$, respectively. The analysis was performed at room temperature (RT) using the focused beam on a randomly oriented sample.

Integral Conversion Electron Mössbauer Spectroscopy (ICEMS) data were recorded at RT in a conventional constant acceleration spectrometer using a ${ }^{57} \mathrm{Co}(\mathrm{Rh})$ source and a parallel plate avalanche counter. All the isomer shifts were referred to the centroid of the spectrum of $\alpha$-Fe at RT.

The dependence of the magnetization on temperature was measured in the range of 20-300 K with an in-plane applied field of 2 kOe using a SQUID magnetometer. Hysteresis loops were measured at 300, 150 and $100 \mathrm{~K}$ with a maximum in-plane applied field of $20 \mathrm{kOe}$. Evolution of angular dependence of RT hysteresis loops with the in-plane applied magnetic field was 
measured by MOKE. Measurements were performed with angular steps of 4.5 degrees and a maximum applied field of $1.25 \mathrm{kOe}$.

\section{Results}

In the present study we first show the results obtained by the different characterization methods, followed by a discussion and the implications on the fabrication of magnetite thin films by PLD.

\subsection{Surface structure}

The deposits were examined by AFM to characterize the surface structure and thickness. For each sample several scans of $2 \times 2 \mu \mathrm{m}^{2}$ were acquired. The values of thickness and roughness of the deposits are presented in Table 1.

Table 1. Thickness, roughness and size of nanostructures of deposits obtained upon irradiation at different wavelengths and substrate temperatures, as determined by AFM measurements.

\begin{tabular}{lllll}
\hline $\begin{array}{l}\text { Laser wavelength } \\
(\mathrm{nm})\end{array}$ & $\begin{array}{l}\text { Substrate } \\
\text { temperature }(\mathrm{K})\end{array}$ & $\begin{array}{l}\text { Thickness } \\
(\mathrm{nm})\end{array}$ & $\begin{array}{l}\text { Roughness } \\
(\mathrm{nm})\end{array}$ & $\begin{array}{l}\text { Nanostructure } \\
\text { size }(\mathrm{nm})\end{array}$ \\
\hline 213 & 750 & $11 \pm 2$ & $6 \pm 1$ & $30-80$ \\
532 & 750 & $43 \pm 2$ & $9 \pm 1$ & $80-120$ \\
1064 & 750 & $117 \pm 20$ & $17 \pm 1$ & $100-150$ \\
1064 & 475 & $128 \pm 14$ & $15 \pm 9$ & $40-90$ \\
1064 & 300 & $23 \pm 2$ & $14 \pm 5$ & $40-90$ \\
\hline \hline
\end{tabular}

Examples of AFM height images of the deposits obtained by irradiation at different wavelengths at a substrate temperature of $750 \mathrm{~K}$ are shown in Fig. 1. Upon irradiation at $213 \mathrm{~nm}$ 
(Fig. 1a) the deposits are constituted by round nanoparticles with sizes of few tens of nanometers. The mean diameter distribution can be fitted by a lognormal distribution with an average size of $60 \mathrm{~nm}$ and a FWHM of $41 \mathrm{~nm}$. In the case of $532 \mathrm{~nm}$ irradiation (Fig. 1b), most of the nanoparticles present a rectangular-like shape with sizes of up to $120 \mathrm{~nm}$. When the deposits are produced under irradiation at $1064 \mathrm{~nm}$ (Fig. 1c), the nanostructures appear as a mixture of cubic and orthogonal prisms with straight edges, with face dimensions of $100-150 \mathrm{~nm}$. The characteristic nanostructure sizes at each wavelength are listed in Table I.
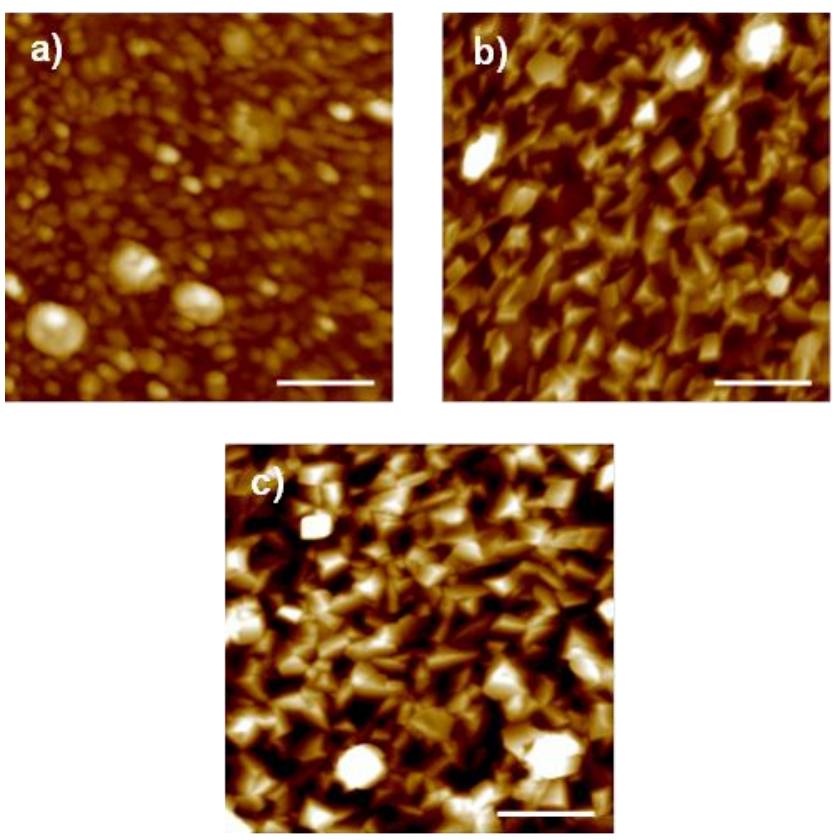

Fig 1. Height AFM images of the deposits obtained at a substrate temperature of $750 \mathrm{~K}$ and upon irradiation at: a) 213 , b) 532 and c) $1064 \mathrm{~nm}$. All the images are $80 \mathrm{~nm}$ high and the scale bar is $500 \mathrm{~nm}$.

Fig. 2a displays an AFM height image of the deposits obtained by irradiation at $1064 \mathrm{~nm}$ at a substrate temperature of $475 \mathrm{~K}$. In this case, and in contrast with the results obtained at $750 \mathrm{~K}$, the deposit contains round nanoparticles with sizes of few tens of nanometers. When the 
substrate is kept at $300 \mathrm{~K}$ (Fig. 2b), the deposit is also constituted by round nanoparticles. Some larger aggregates, of up to $200 \mathrm{~nm}$, can be observed in both cases.
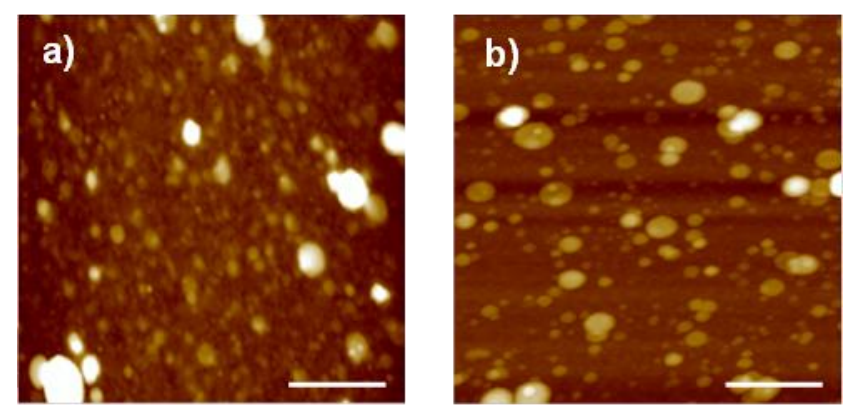

Fig. 2. Height AFM images of the deposits obtained upon irradiation at $1064 \mathrm{~nm}$ with the substrate at a temperature of: a) 475 and b) $300 \mathrm{~K}$. All the images are $80 \mathrm{~nm}$ high and the scale bar is $500 \mathrm{~nm}$.

The results herein indicate that the laser wavelength and substrate temperature exert a strong influence on the morphology and dimensions of the nanostructured films produced by ns PLD from hematite targets.

\subsection{Surface crystallinity}

Fig. 3 presents XRD patterns of samples grown on a substrate heated at $750 \mathrm{~K}$. The XRD peaks are located at $30.1^{\circ}, 35.4^{\circ}, 37.1^{\circ}, 43.1^{\circ}, 53.4^{\circ}, 57^{\circ}$, and $62.6^{\circ}$ and are assigned to the $\mathrm{Fe}_{3} \mathrm{O}_{4}$ (220), (311), (222), (400), (422), (511) and (440) diffraction planes, respectively. This pattern is indicative of the polycrystalline nature of the grown magnetite (Joint Committee on Powder Diffraction Standards Card No. 88-0866). The additional broad band centered at $69.2^{\circ}$ (not shown completely) corresponds to the diffraction line (400) of the Si substrate. No diffraction peaks corresponding to hematite are observed. For the deposits fabricated at $213 \mathrm{~nm}$ (Fig. 3a), the small peak observed at $44.7^{\circ}$ corresponds to the (110) plane of metallic iron. 
The average crystalline domain size of the deposits can be estimated using the Scherrer law, $D=0.9 \lambda / B \cos \theta_{B}$, where $\lambda$ is the X-ray wavelength $(\mathrm{Cu} \mathrm{K} \alpha=0.154 \mathrm{~nm})$ and $B$ is the full width at half-maximum (in radians) of the diffraction peak. The Scherrer law does not include peak broadening due to stress/strain, which is important in the case of thin films, and therefore the calculated crystalline domain sizes are over/subestimated. Nevertheless, the obtained values can be used to assess the influence of deposition conditions on the crystallinity of the deposits. The calculated nanocrystalline domain sizes are $24 \pm 3,37 \pm 6$ and $44 \pm 4 \mathrm{~nm}$, for 213 , 532 and $1064 \mathrm{~nm}$ irradiation, respectively, showing larger size upon increasing the deposition wavelength.

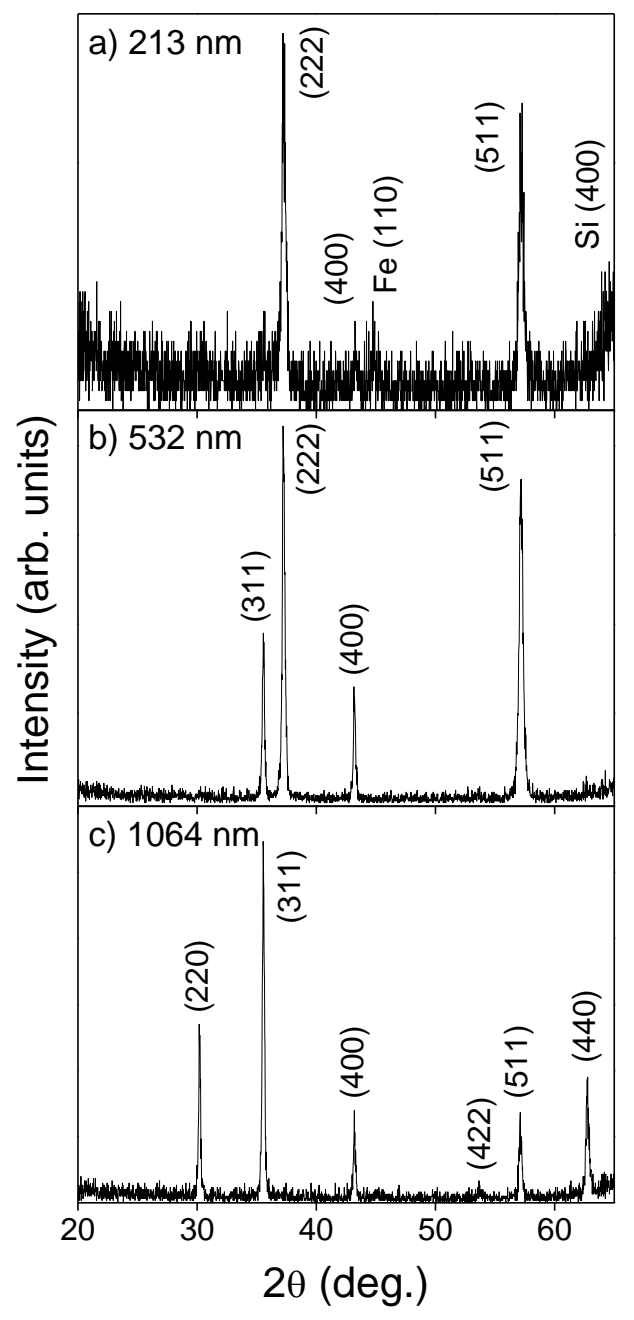


Fig. 3. XRD patterns of nanostructured films grown by heating the substrate at $750 \mathrm{~K}$ and upon irradiation at: a) 213, b) 532 and c) $1064 \mathrm{~nm}$. The labels of the diffraction peaks correspond to magnetite unless other material is indicated.

Samples grown by irradiation at $1064 \mathrm{~nm}$ at different substrate temperatures were also analyzed by XRD. The XRD pattern obtained from the film grown at $475 \mathrm{~K}$ (Fig. 4a) reveals that only polycrystalline magnetite is obtained. The diffractogram recorded from the sample grown with the substrate temperature at $300 \mathrm{~K}$ (Fig. 4b) shows broad peaks and it resembles that of a poorly crystalline material either with small particle size and/or abundant structural defects. The main peaks correspond to the (220), (311), and (400) diffraction peaks of magnetite. Despite the poor signal-to-noise ratio of the diffraction pattern, the small peaks at around $36^{\circ}$ and $42^{\circ}$ could be associated with the (111) and (200) diffraction planes of wüstite $\left(\mathrm{Fe}_{1-\mathrm{x}} \mathrm{O}\right)$. The nanocrystalline domain sizes estimated using the Scherrer law are as small as $13 \pm 3$ and $9 \pm 2 \mathrm{~nm}$ for the samples grown at 475 and $300 \mathrm{~K}$, respectively, by comparison with $44 \pm 4 \mathrm{~nm}$ determined for the film deposited at $750 \mathrm{~K}$. 


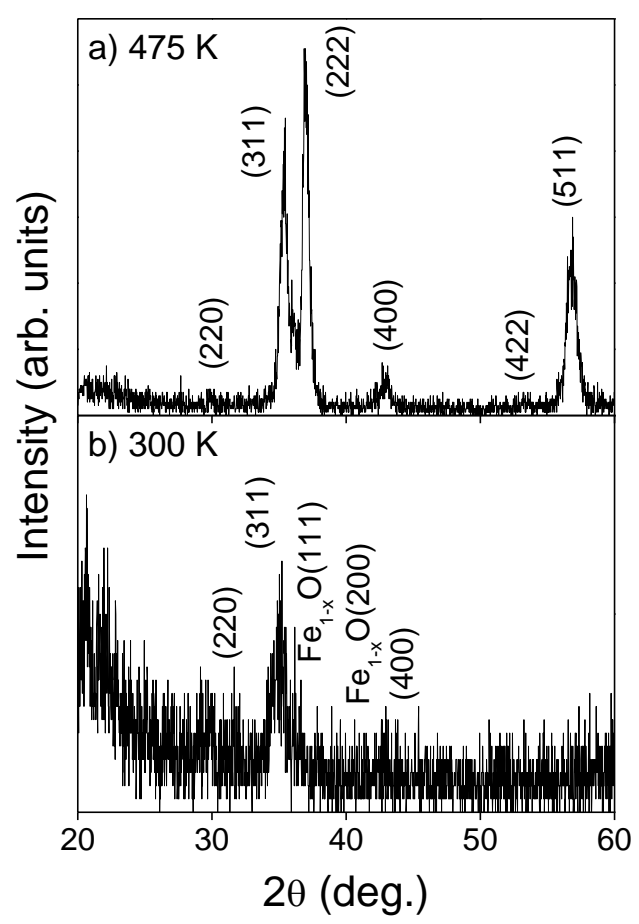

Fig. 4. XRD pattern of nanostructured films grown at $1064 \mathrm{~nm}$, with the substrate at a temperature of: a) 475 and b) $300 \mathrm{~K}$. The labels of the diffraction peaks correspond to magnetite unless other material is indicated.

\subsection{Micro-Raman spectroscopy}

The XRD patterns of magnetite and maghemite $\left(\gamma-\mathrm{Fe}_{2} \mathrm{O}_{3}\right)$ differ only for the presence of few diffraction peaks with intensity lower than $5 \%$. Thus, the absence of these low-intensity peaks from the X-ray diffractograms shown in Fig. 3 and Fig. 4, cannot be considered as a proof of the formation of single, pure, stoichiometric magnetite nanostructured films. An alternative technique is the use of micro-Raman spectroscopy, considered a powerful tool to distinguish the different iron oxides phases,[42] which differ in vibrational frequencies. Micro-Raman spectra of the deposits fabricated on substrates heated at $750 \mathrm{~K}$ at different irradiation wavelengths are shown in Fig. 5. Although some bands of magnetite overlap with those corresponding to the $\mathrm{Si}$ 
substrate, the characteristic magnetite features at $668,538,303$, and $193 \mathrm{~cm}^{-1}$ were observed under all deposition conditions. The pronounced magnetite feature at $668 \mathrm{~cm}^{-1}$, attributed to the $\mathrm{A}_{1 \mathrm{~g}}$ mode, is clearly visible in all the spectra, whereas the features at $303 \mathrm{~cm}^{-1}$ and $538 \mathrm{~cm}^{-1}$, assigned to the $\mathrm{E}_{\mathrm{g}}$ and $\mathrm{T}_{2 \mathrm{~g}}$ modes respectively, are weak and overlap with the 519 and $303 \mathrm{~cm}^{-1}$ bands of the substrate. A very low intensity feature at $193 \mathrm{~cm}^{-1}$, also assigned to the $\mathrm{T}_{2 \mathrm{~g}}$ mode, can be observed in the samples prepared upon 532 and $1064 \mathrm{~nm}$ irradiation. For the sample prepared at $213 \mathrm{~nm}$ the spectrum displays the substrate features, clearly observed at 623,519 , 435 , and $300 \mathrm{~cm}^{-1}$ and a broad band in the range of $930-990 \mathrm{~cm}^{-1}$. There is no observable peak shift in the Raman spectra, suggesting the absence of contributions from non-equivalent sites in the magnetite structure.[42]

The micro-Raman spectra of samples fabricated upon irradiation at $1064 \mathrm{~nm}$ on substrates at 475 and at $300 \mathrm{~K}$, are rather similar (not shown), with all bands corresponding to magnetite. We could not verify the presence of wüstite in the deposits by micro-Raman spectroscopy probably due to the low intensity of the corresponding feature (at $650 \mathrm{~cm}^{-1}$ ), consequence of its reduced content (as suggested by XRD), in combination with overlapping of the magnetite band at $668 \mathrm{~cm}^{-1}$.

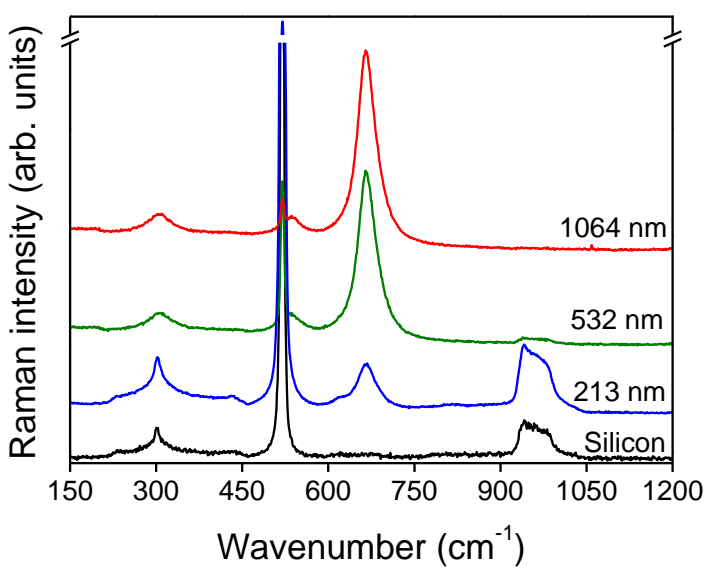


Fig. 5. Micro-Raman spectra of $\mathrm{Fe}_{3} \mathrm{O}_{4}$ nanostructured films grown by PLD at 213, 532, and 1064 $\mathrm{nm}$, as indicated, by heating the substrate at $750 \mathrm{~K}$. Micro-Raman spectrum of the silicon substrate is shown for comparison.

\subsection{Integral Conversion Electron Mössbauer Spectroscopy (ICEMS)}

Magnetite presents an inverse spinel structure, with the $\left[\mathrm{Fe}^{3+}\right]_{\mathrm{A}}\left[\mathrm{Fe}^{3+} \mathrm{Fe}^{2+}\right]_{\mathrm{B}} \mathrm{O}_{4}$ formula, where A indicates tetrahedral sites, and B octahedral sites. At RT there is fast electron hopping between the $\mathrm{Fe}$ cations at the octahedral sites, giving an average +2.5 valence in Mössbauer spectroscopy.[43] Each different iron cation $\left(\mathrm{Fe}^{3+}\right.$ at tetrahedral sites and $\mathrm{Fe}^{2.5^{+}}$at octahedral sites) gives rise to a different sextet component. In both cases, the Mössbauer quadrupole shift $(2 \varepsilon)$, related to the distortion of the atomic environment from the ideal cubic symmetry, is close to 0 . The hyperfine magnetic field is smaller for the octahedral site, reflecting the smaller magnetic moment of $\mathrm{Fe}^{2.5+}{ }_{\mathrm{B}}$. For stoichiometric magnetite,[43] the ratio between both components $\left(S_{B} / S_{A}\right)$ is very close to $2(1.9)$ with $\delta_{A}=0.29 \mathrm{mms}^{-1}, 2 \varepsilon_{A}=0.00 \mathrm{mms}^{-1}, H_{A}=49 \mathrm{~T}$; $\delta_{B}=0.66 \mathrm{mms}^{-1}, 2 \varepsilon_{A}=0.00 \mathrm{mms}^{-1}$, and $H_{B}=46 \mathrm{~T}$, where $\delta$ is the isomer shift and $H$ the hyperfine magnetic field. Mössbauer spectra recorded from the different samples are presented in Fig. 6 and the parameters obtained from the fits are collected in Table 2. 
Table 2. Mössbauer parameters obtained from the fit of spectra recorded from films deposited at the indicated conditions. $\delta$ and $2 \varepsilon$ are the isomer and quadrupole shifts, respectively, and $H$ the hyperfine field.

\begin{tabular}{|c|c|c|c|c|c|c|c|}
\hline $\begin{array}{l}\text { Irradiation } \\
\text { conditions }\end{array}$ & Component & $\begin{array}{l}\delta \\
\left(\mathrm{mm} \mathrm{s}^{-1}\right)\end{array}$ & $\begin{array}{l}2 \varepsilon \\
\left(\mathrm{mm} \mathrm{s}^{-1}\right)\end{array}$ & $\begin{array}{l}\mathrm{H} \\
(\mathrm{T})\end{array}$ & $\begin{array}{l}\text { Area } \\
(\%)\end{array}$ & $\begin{array}{l}\mathrm{A}_{2} / \mathrm{A}_{3} \\
\text { area ratio }\end{array}$ & $\begin{array}{l}\mathrm{S}_{\mathrm{B}} / \mathrm{S}_{\mathrm{A}} \text { area } \\
\text { ratio }\end{array}$ \\
\hline $213 \mathrm{~nm}$ & Doublet & 0.27 & 0.49 & - & 2 & - & \\
\hline \multirow[t]{3}{*}{$750 \mathrm{~K}$} & Sextet A & 0.27 & 0.00 & 48.7 & 37 & 3.00 & $1.54 \pm 0.22$ \\
\hline & Sextet B & 0.66 & 0.00 & 45.4 & 57 & & \\
\hline & $\alpha-\mathrm{Fe}$ & -0.02 & -0.12 & 33.0 & 4 & 3.00 & \\
\hline $532 \mathrm{~nm}$ & Sextet A & 0.27 & 0.00 & 48.7 & 37 & 2.3 & $1.70 \pm 0.17$ \\
\hline $750 \mathrm{~K}$ & Sextet B & 0.65 & 0.00 & 45.4 & 63 & & \\
\hline $1064 \mathrm{~nm}$ & Sextet A & 0.26 & -0.02 & 49.3 & 35 & 2.75 & $1.85 \pm 0.15$ \\
\hline $750 \mathrm{~K}$ & Sextet B & 0.67 & 0.00 & 46.1 & 65 & & \\
\hline $1064 \mathrm{~nm}$ & Sextet A & 0.25 & -0.04 & 48.4 & 33 & 1.00 & $2.00 \pm .0 .15$ \\
\hline $475 \mathrm{~K}$ & Sextet B & 0.64 & -0.04 & 45.4 & 67 & & \\
\hline $1064 \mathrm{~nm}$ & Sextet A & 0.26 & 0.02 & 46.6 & 17 & $(*)$ & $(*)$ \\
\hline \multirow[t]{4}{*}{$300 \mathrm{~K}$} & Sextet B & 0.59 & -0.08 & 43.9 & 38 & & \\
\hline & Fe $2.5+$ & 0.61 & 0,06 & 39.6 & 30 & & \\
\hline & $\mathrm{Fe}_{1-\mathrm{x}} \mathrm{O}$ & 0.68 & 1.67 & ---- & 11 & & \\
\hline & $\mathrm{Fe}_{1-\mathrm{x}} \mathrm{O}$ & 1.02 & 0.89 & ---- & 4 & & \\
\hline
\end{tabular}

(*)Not calculated due to the complexity of the spectrum 


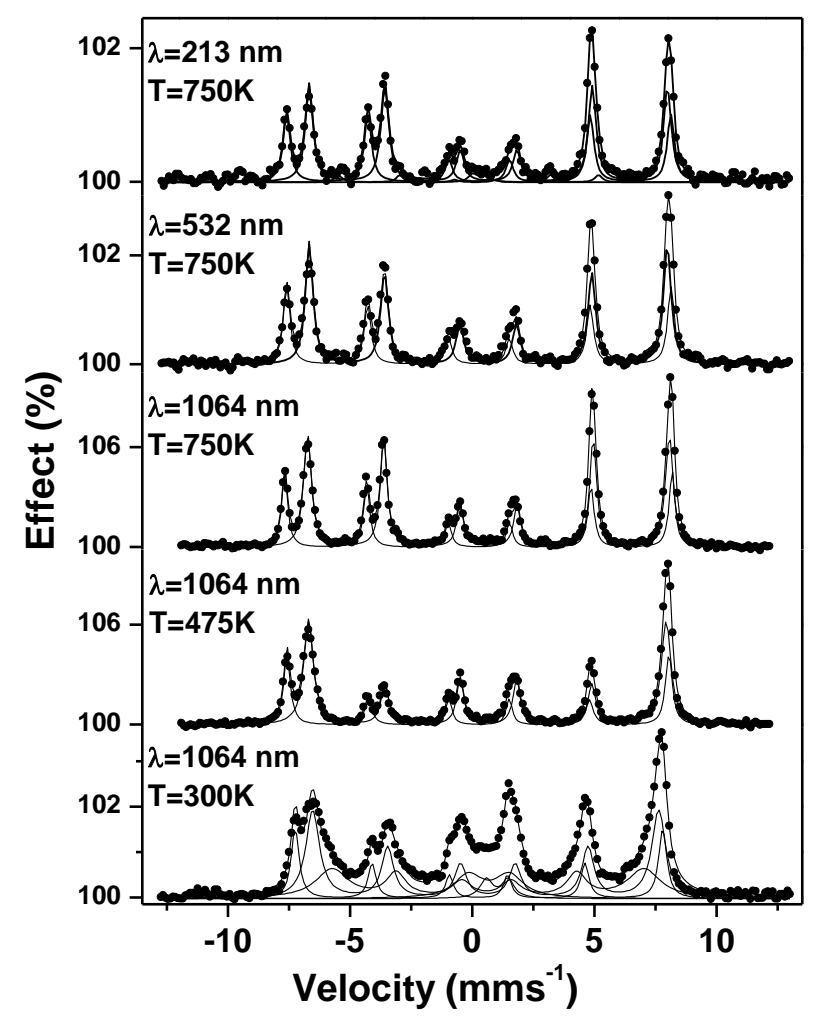

Fig. 6. ICEMS spectra recorded from nanostructured films deposited at the indicated irradiation conditions.

For the deposits prepared at $750 \mathrm{~K}$, with the exception of the film prepared at $213 \mathrm{~nm}$, where a small $(<3 \%)$ additional contribution due to metallic iron is also observed, the spectra contain only the two sextets which are characteristic of magnetite. The ratio between the $S_{B}$ and $S_{A}$ sextets, which should be 1.9 for stoichiometric magnetite, is lower than this value for the samples prepared under UV irradiation $(213 \mathrm{~nm})$. Non-stoichiometric magnetite can be formulated, assuming the existence of vacancies only in the octahedral sites of its spinel-related structure, as $\mathrm{Fe}^{3+}\left[\mathrm{Fe}^{2+}{ }_{1-3 \mathrm{x}} \mathrm{Fe}^{3+}{ }_{1+2 \mathrm{x}} \square_{\mathrm{x}}\right] \mathrm{O}_{4}$ or, taking into account the $2.5+$ average oxidation state of iron in the octahedral sites, as $\mathrm{Fe}^{3+}\left[\mathrm{Fe}^{2.5+}{ }_{2(1-3 \mathrm{x})} \mathrm{Fe}^{3+}{ }_{5 \mathrm{x}} \mathrm{D}_{\mathrm{x}}\right] \mathrm{O}_{4}$, where $0 \leq \mathrm{x} \leq 0.33$. The end member of the series, corresponding to $\mathrm{x}=0.33$, is maghemite, $\mathrm{Fe}^{3+}\left[\mathrm{Fe}^{3+}{ }_{5 / 3} \square_{\mathrm{x}}\right] \mathrm{O}_{4}$. This implies that a $S_{B} / S_{A}$ area 
ratio lower than 1.6 does not make sense within the context of a single phase non-stoichiometric magnetite. The only way to explain the obtained ratio (1.54) in the film prepared at $213 \mathrm{~nm}$ (Table 2), is to assume the presence of a small, additional amount of maghemite. The spectrum of maghemite overlaps with that of the tetrahedral component of magnetite and to discern both is, in practical terms, impossible,[43] especially if the amount of maghemite is small, as in the present case. This may be probably the reason why maghemite goes undetected in the microRaman experiments.

The $S_{B} / S_{A}$ ratio approaches 1.9 , i.e. the pure stoichiometric value, as the irradiation wavelength increases, clearly showing that the stoichiometry of the deposited magnetite depends on the wavelength used during PLD: larger wavelengths induce the formation of almost stoichiometric magnetite while shorter wavelengths increase the amount of $\mathrm{Fe}^{3+}$. We can rule out the presence of iron oxides such as hematite, which should show only one sextet with a $H$ of about $52 \mathrm{~T}$ and a $2 \varepsilon$ of $-0.20 \mathrm{mms}^{-1}$.

Mössbauer spectroscopy also gives information about the average magnetization direction. The spectra have been acquired in the as-grown films. The ratio between the $2^{\text {nd }}$ and $3^{\text {rd }}$ component of each sextet $\left(A_{2} / A_{3}\right.$ area ratio) should be 2 for random magnetization orientation, and $A_{2} / A_{3}$ larger than 2 should implies a tendency of in-plane magnetization orientation. The obtained results indicate that the film fabricated at $1064 \mathrm{~nm}$ and $475 \mathrm{~K}$ has out-of-plane orientation whereas all other films have in-plane orientation, without showing a clear trend with irradiation wavelength. The values observed might be related to changes in the microcrystalline texture of the films.

It is observed that the films fabricated at $1064 \mathrm{~nm}$ and at a substrate temperature of $475 \mathrm{~K}$ (Fig. 6) are exclusively constituted by magnetite although the spectral lines appear to be broader than in the spectrum of the sample prepared at $750 \mathrm{~K}$, what would suggest a slightly larger 
degree of structural inhomogeneities. However, the $S_{B} / S_{A}$ area ratio is 2.0 , i.e., as it would be expected for stoichiometric magnetite. The spectrum recorded from the film deposited at $300 \mathrm{~K}$ (Fig. 6) is also compatible with the formation of magnetite. However, in addition to the two narrow sextets corresponding to the A and B sites of magnetite there is a third broad magnetic component with $\delta=0.61 \mathrm{mms}^{-1}$, similar to that shown by the $\mathrm{Fe}^{2.5+}$ component but with a much smaller $H(39.6 \mathrm{~T})$. The results are compatible with incomplete structural and/or magnetic order at the octahedral sites of the spinel-related structure of $\mathrm{Fe}_{3} \mathrm{O}_{4}$. Besides this, the hyperfine magnetic field values of the narrower A and B sextets are smaller than those characteristic of bulk magnetite $\left(H_{A}=49.0 \mathrm{~T}, H_{B}=46.0 \mathrm{~T}\right)$. Taken together, the results indicate that the film deposited at $300 \mathrm{~K}$ is of poorly crystalline character and/or is composed by small particles. The spectrum also shows (Fig. 6) paramagnetic contributions (15\%) in its central part which have been fitted to two doublets. The Mössbauer parameters of these two doublets $\left(\delta=0.68 \mathrm{mms}^{-1}\right.$, $\Delta=1.67 \mathrm{mms}^{-1} ; \delta=1.02 \mathrm{mms}^{-1}, \Delta=0.89 \mathrm{mms}^{-1}$ ) could be associated to the presence of $\mathrm{Fe}_{1-\mathrm{x}} \mathrm{O}$.

\subsection{Magnetic characterization}

A plot of the magnetization as a function of temperature for the deposits fabricated at a substrate temperature of $750 \mathrm{~K}$ and wavelengths of 213, 532 and $1064 \mathrm{~nm}$ is shown in Fig. 7. Magnetization was measured on warming in an in-plane magnetic field of $2 \mathrm{kOe}$ after zero field cooling. A sudden jump in magnetization can be observed when increasing the temperature for all the films as a result of the change in the crystal structure from the low temperature monoclinic phase to the high temperature cubic one. The temperature at which this transition occurs, the Verwey temperature $\left(T_{V}\right)$, has been determined for each film by taking the maximum slope of the magnetization. 


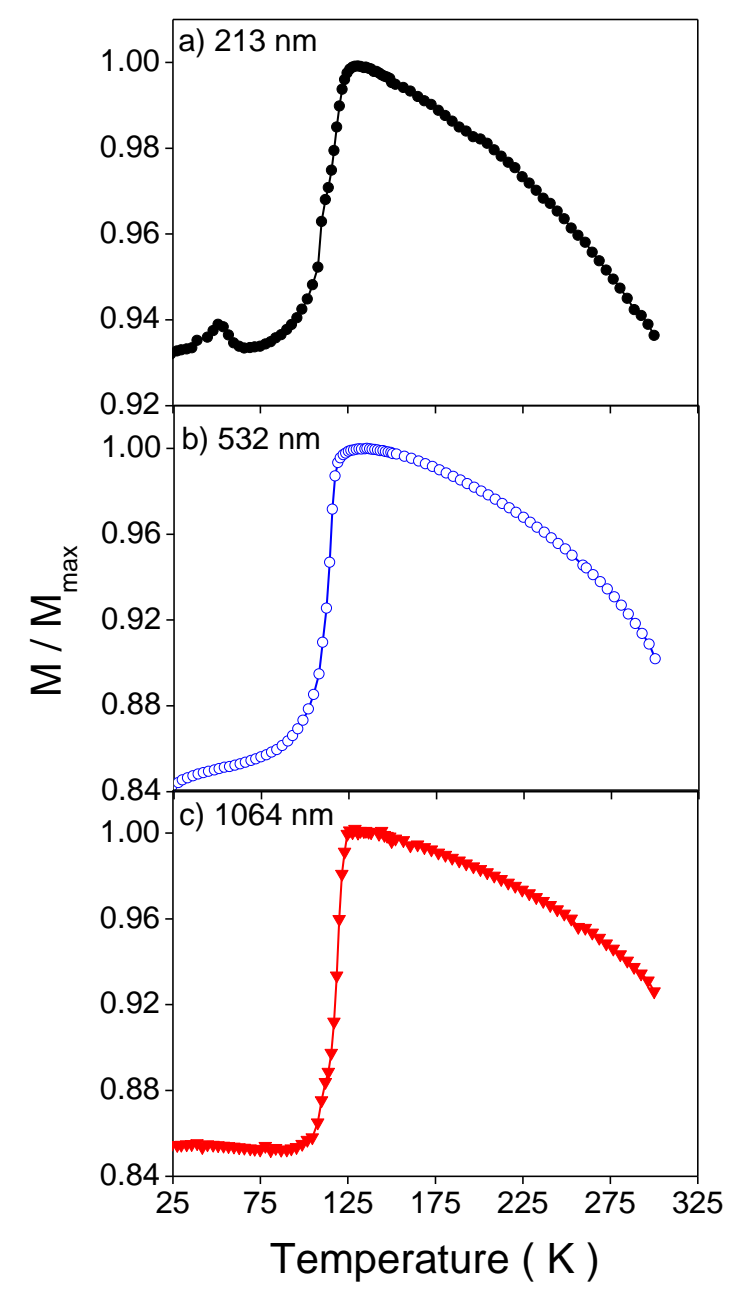

Fig. 7. Dependence of the magnetization on temperature (warming curves) with an in-plane applied field of $2 \mathrm{kOe}$ for magnetite films fabricated at a substrate temperature of $750 \mathrm{~K}$ and a deposition wavelength: a) $213 \mathrm{~nm}$, b) $532 \mathrm{~nm}$, and c) $1064 \mathrm{~nm}$.

A clear Verwey transition is measured at about $110 \mathrm{~K}$ and $114 \mathrm{~K}$ for films deposited at 213 and $532 \mathrm{~nm}$, respectively. The Verwey transition for both samples is smeared out possibly due to the interfacial strain induced in the films. The film deposited at $1064 \mathrm{~nm}$ (thickest film) with $T_{V}=119 \mathrm{~K}$, shows the sharpest increase of magnetization in the smallest temperature range among the complete series. 
Demagnetization curves measured at three different temperatures (300, 150 and $100 \mathrm{~K})$ for samples prepared at a substrate temperature of $750 \mathrm{~K}$ and wavelengths of 213,532 and $1064 \mathrm{~nm}$ are shown in Fig. 8. A decrease in coercivity is observed at all measurement temperatures under study with increasing deposition wavelength (Fig. 8d). At $300 \mathrm{~K}$, the largest coercivity (415 Oe) is obtained for the magnetite film prepared at $213 \mathrm{~nm}$ by comparison with a minimum value of 232 Oe achieved for the magnetite film grown at $1064 \mathrm{~nm}$. At $100 \mathrm{~K}$, these films achieve coercivity values of 742 Oe and 688 Oe, respectively.
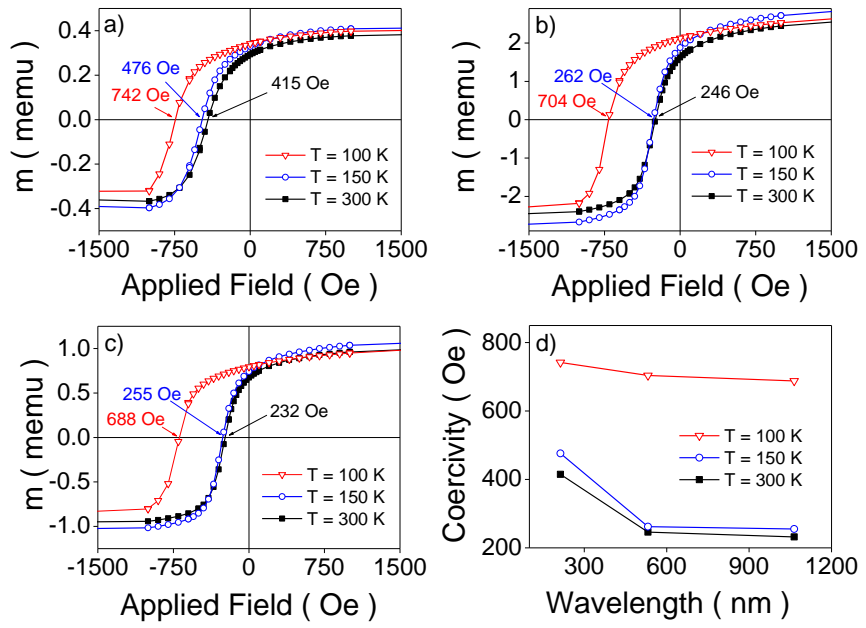

Fig. 8. Demagnetization curves at the indicated temperatures for magnetite films fabricated at a substrate temperature of $750 \mathrm{~K}$ and a deposition wavelength of: a) 213 , b) 532, and c) $1064 \mathrm{~nm}$. d) Evolution of coercivity vs deposition wavelength measured at different temperatures (lines are guide to the eye).

The large increase in coercivity at $T<T_{V}$ (Fig. $8 \mathrm{~d}$ ) is a well known result due to the abrupt change in the magnetocrystalline and magnetostriction constants when magnetite transforms from the cubic to the monoclinic phase.[31] The large magnitude of the low temperature monoclinic magnetocrystalline anisotropy constants, compared to the cubic ones, and the abrupt 
changes in the magnetostriction constant are considered to be the dominant factors controlling the demagnetizing behavior below $T_{V}$.

Fig. 9 shows the dependence of the magnetization on temperature for magnetite films grown at $1064 \mathrm{~nm}$ and at substrate temperatures of 475 and $300 \mathrm{~K}$, under the same measurement conditions used to obtain the curves displayed in Fig. 7. The decreased microstructural and morphological quality of these films is evident in the magnetization curves with a decreased $T_{V}$ of about $109 \mathrm{~K}$ and even the absence of a well defined $T_{V}$ for the films prepared at 475 and $300 \mathrm{~K}$, respectively.
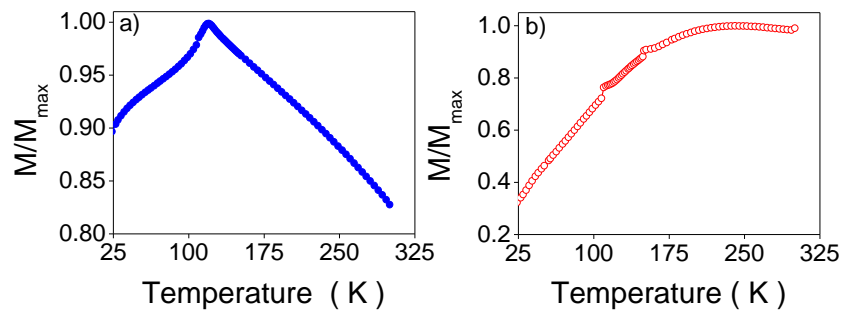

Fig. 9. Dependence of the magnetization on temperature (warming curves) with an in-plane applied field of $2 \mathrm{kOe}$ for magnetite films fabricated with a deposition wavelength of $1064 \mathrm{~nm}$ at substrate temperatures of: a) $475 \mathrm{~K}$ and b) $300 \mathrm{~K}$.

The above mentioned features for both films correlate well with the hysteresis loops shown in Fig. 10. A decreased substrate temperature of $475 \mathrm{~K}$ results in a RT coercivity value (255 Oe) higher than that obtained for the film grown at $750 \mathrm{~K}(232 \mathrm{Oe})$, shown in Fig. $8 \mathrm{~d}$, as a consequence of a finer microstructure consisting of a larger density of intergranular and defective regions. 

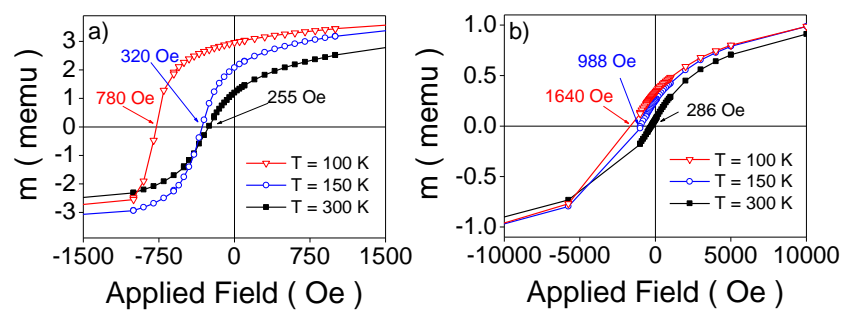

Fig. 10. Demagnetization curves at the indicated temperatures for magnetite films fabricated with a wavelength of $1064 \mathrm{~nm}$ at different substrate temperatures of: a) $475 \mathrm{~K}$ and b) $300 \mathrm{~K}$.

The angular dependence of the films has been studied by MOKE by recording hysteresis loops with angular variation (0-360 degrees with 4.5 degrees steps) in the in-plane orientation of the applied external magnetic field. Fig. 11 shows representative hysteresis loops measured at angular intervals of 90 degrees for a magnetite film deposited at a wavelength of $532 \mathrm{~nm}$ and a substrate temperature of $750 \mathrm{~K}$. For all the irradiation wavelengths the loops were identical independently of the in-plane angle indicating that the magnetite films grown at different deposition wavelengths are magnetically isotropic.

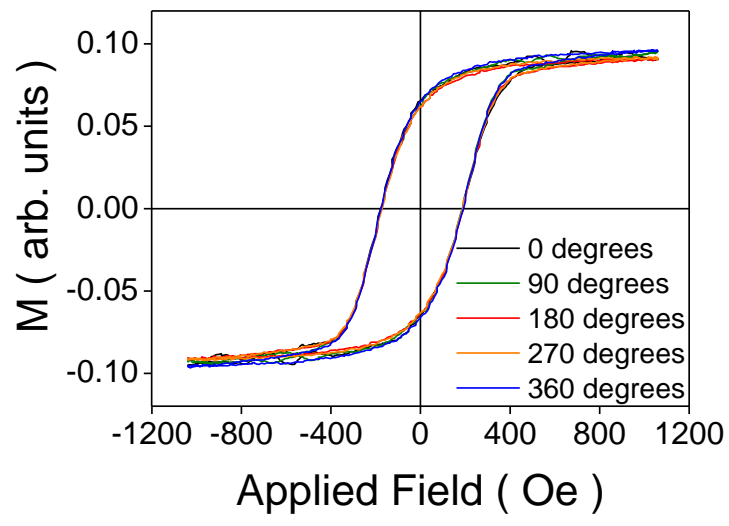

Fig. 11. Hysteresis loops measured by MOKE at different angles of the in-plane magnetic field for a magnetite film grown with a wavelength of $532 \mathrm{~nm}$ and a substrate temperature of $750 \mathrm{~K}$. 


\section{Discussion}

The properties of the nanostructured films prepared by PLD on Si substrates follow clear trends with laser irradiation wavelengths and substrate temperature, with consistency between the results obtained by the different and complementary techniques used in this study. AFM indicates that, while a shorter wavelength produces a roughly more uniform film, the shape of the grains in such film is less defined, showing less developed facets than for longer wavelengths. Deposition at a lower substrate temperature also reduces the particle size but again at the expense of less defined facets. XRD results correlate with the AFM results: the most crystalline films are the ones grown at elevated temperature and under longer irradiation wavelength. XRD results also show that the films are composed of magnetite (although maghemite could not be ruled out due to overlapping of reflection peaks with the former). The micro-Raman spectra prove the presence of magnetite,[44] and are conclusive on the absence of any relevant features indicating a significant presence of either maghemite or hematite. The ratio of the two cation sites from Mössbauer spectroscopy approaches the magnetite stoichiometric value as the irradiation wavelength increases whilst shorter wavelengths increase the amount of $\mathrm{Fe}^{3+}$

The Verwey transition is known to be very sensitive to the stoichiometry.[11, 12] While the sharper Verwey transition observed for the film grown under the longer wavelength of $1064 \mathrm{~nm}$ can be attributed to the larger thickness of that film, the transition temperature corresponds to high quality magnetite. In fact, lowering the temperature of the substrate during deposition, even for films of the same thickness, results in a less defined Verwey transition (smoothed out magnetization curves, decreased Verwey temperature). The Verwey transition disappears for the $300 \mathrm{~K}$ grown film, in agreement with the very wide sextets observed by Mössbauer spectroscopy 
which are indicative of small particle sizes or poor crystallinity. Furthermore, for the less crystalline films, an increased number of intergranular and or disordered regions are expected which would pin down the magnetic domains increasing the coercivity[31] as experimentally observed. The lowest coercivity of the higher substrate temperature and irradiation wavelength correlates well with that of bulk magnetite.

The changes in film quality can be discussed in relation to the initial laser-target interaction, the subsequent plume expansion and the nucleation processes that take place on the substrate. In the case of ns laser ablation, the main processes induced during target irradiation are normal vaporization, phase explosion and subsurface heating. $[45,46]$ The tendency to produce smaller nanoparticles with a narrower size distribution at shorter wavelengths as observed herein has also been reported previously for other solid target materials.[4-7] We note, though, that if the goal is to obtain a crystalline film (as opposed to a film constituted by well defined nanoparticles) the presence of smaller nanoparticles would indicate that these are not the ideal growing conditions. Considering the linear absorption coefficients of the target, $\alpha_{213} \approx 8 \times 10^{5} \mathrm{~cm}^{-1}$, $\alpha_{532} \approx 2 \times 10^{4} \mathrm{~cm}^{-1}$ and $\alpha_{1064} \approx 1 \times 10^{3} \mathrm{~cm}^{-1}$,[47] the heated subsurface layer thickness is only $12 \mathrm{~nm}$ at $213 \mathrm{~nm}$, while it amounts to around $500 \mathrm{~nm}$ for $532 \mathrm{~nm}$ and $10 \mu \mathrm{m}$ for $1064 \mathrm{~nm}$. Therefore, the experimentally observed tendency to produce smaller nanoparticles at $213 \mathrm{~nm}$ irradiation is related to the smaller size of the ejected species (ions, atoms and small atomic clusters) produced in a reduced volume during the relaxation of the ablated target. Further characterization of the energy profile of the emitted species particles from the target as a function of wavelength remains the subject of future studies.

One reason for the complete prevalence in previous works of the use of UV wavelength in the preparation of magnetite films by PLD is the avoidance of target particulate ejection. As the PLD 
growth from a hematite target is not congruent, a simple test for such process is the detection of hematite in the grown films. We have not detected any sign of the presence of hematite in the films prepared at the various experimental conditions explored by any of the techniques employed in the present work: XRD, micro-Raman, and Mössbauer spectroscopy.

The role of temperature can be understood considering the mobility of the deposited material on the surface, which is favored by increasing the substrate temperature. This dependence indicates that the thermal annealing of the small amount of material deposited at each laser pulse (a thousand of a monolayer) is also a crucial processing parameter to promote smooth growth, and thus, well defined magnetite crystallites. Work is in progress to use the present parameters in the growth of single crystalline magnetite by the choice of the appropriate substrates.

\section{Conclusions}

Magnetite nanostructured deposits on $\mathrm{Si}$ substrates were obtained by PLD from sintered hematite targets at the wavelengths of 213,532 , and $1064 \mathrm{~nm}$ at different substrate temperatures, from a Q-switched Nd:YAG laser. Detailed morphological, microstructural and compositional characterization of the films has been carried out by means of AFM, XRD, micro-Raman and Mössbauer spectroscopy, while SQUID and MOKE techniques have been used for magnetic characterization. Except for the shorter wavelength, the obtained deposits are almost stoichiometric magnetite. In particular no hematite is detected, independently of the laser irradiation wavelength, indicating that hematite particles clusters are not present in the ablation plume. The observed trend of stoichiometry improvement of deposits as the laser wavelength increases, and the importance of the latter parameter in the crystalline and magnetic properties of the deposits, provide the possibility of tailoring the properties of magnetic nanostructured films 
formed by PLD. The best quality deposits, having the right magnetite stoichiometry as indicated by Mössbauer spectroscopy, with a Verwey transition at $119 \mathrm{~K}$ and a coercivity of 232 Oe at RT, were grown by ablation at $1064 \mathrm{~nm}$ and at a substrate temperature of $750 \mathrm{~K}$. Thin films fabricated at these conditions simultaneously present magnetite nanoparticles of geometrical shape with well defined edges, in a mixture of cubic and orthogonal prisms, with sizes in the range 100-150 nm. As an alternative to conventional UV ns PLD, the use of IR PLD, together with the use of self-prepared hematite sintered targets, enhances the quality of magnetite thin films and allows preparing low-cost pure magnetite nanostructured thin films for different applications.

\section{Acknowledgements}

We acknowledge funding under Projects CTQ2010-15680, CONSOLIDER CSD2007-00058 and CSD2009-00013 (MICINN) and MAT2012-3804-C04-0, MAT2011-25598 and MAT201127470-C02-02 (MINECO). E.R, A.B., M.O., M.S. and M.M. gratefully thank financial support from Ramón y Cajal Programme (RYC-2011-08069 and RYC-2007-01727), CSIC contract, Geomateriales (CAM, S2009/Mat-1629) and MICINN FPI Programme, respectively. A.B., F.J.P., and M.G.-H. gratefully thank funding under EU-FP7 NANOPYME Project (No. 310516). We are grateful to Prof. T. A. Ezquerra and M. V. Cañamares (IEM, CSIC) for the use of the AFM system and micro-Raman measurements, respectively, and M. Juanco (ICA, CSIC) for XRD measurements. 


\section{References}

[1] D.B. Chrisey, G.K. Hubler, Pulsed Laser Deposition of Thin Films, John Wiley \& Sons, 1994.

[2] M.N. Ashfold, F. Claeyssens, G.M. Fuge, S.J. Henley, Pulsed laser ablation and deposition of thin films, Chem. Soc. Rev., 33 (2004) 23-31.

[3] R. Eason, Pulsed Laser Deposition of Thin Films: Applications-Led Growth of Functional Materials, John Wiley \& Sons, 2006.

[4] V. Craciun, D. Craciun, Evidence for volume boiling during laser ablation of single crystalline targets, Appl. Surf. Sci., 138-139 (1999) 218-223.

[5] G. Koren, A. Gupta, R.J. Baseman, M.I. Lutwyche, R.B. Laibowitz, Laser wavelength dependent properties of $\mathrm{YBa} 2 \mathrm{Cu} 3 \mathrm{O} 7-\delta$ thin films deposited by laser ablation, Appl. Phys. Lett., 55 (1989) 2450-2452.

[6] M. Sanz, M. Walczak, M. Oujja, A. Cuesta, M. Castillejo, Nanosecond pulsed laser deposition of $\mathrm{TiO} 2$ : nanostructure and morphology of deposits and plasma diagnosis, Thin Solid Films, 517 (2009) 6546-6552.

[7] M. Sanz, M. López-Arias, E. Rebollar, R. De Nalda, M. Castillejo, Laser ablation and deposition of wide bandgap semiconductors: Plasma and nanostructure of deposits diagnosis, J. Nanopart. Res., 13 (2011) 6621-6631.

[8] M.I. Katsnelson, V.Y. Irkhin, L. Chioncel, A.I. Lichtenstein, R.A. de Groot, Half-metallic ferromagnets: From band structure to many-body effects, Rev. Mod. Phys., 80 (2008) 315-378.

[9] R.M. Cornell, U. Schwertmann, Introduction to the Iron Oxides, in: The Iron Oxides, WileyVCH Verlag GmbH \& Co. KGaA, 2004, pp. 1-7.

[10] E.J.W. Verwey, Electronic conduction of magnetite (Fe $3 \mathrm{O} 4$ ) and its transition point at low temperatures [5], Nature, 144 (1939) 327-328.

[11] F. Walz, The Verwey transition - a topical review, J. Phys.: Condens. Matter, 14 (2002) R285.

[12] J. García, G. Subías, The Verwey transition - a new perspective, J. Phys.: Condens. Matter, 16 (2004) R145.

[13] G.Q. Gong, A. Gupta, G. Xiao, W. Qian, V.P. Dravid, Magnetoresistance and magnetic properties of epitaxial magnetite thin films, Phys. Rev. B, 56 (1997) 5096-5099.

[14] D.T. Margulies, F.T. Parker, F.E. Spada, R.S. Goldman, J. Li, R. Sinclair, A.E. Berkowitz, Anomalous moment and anisotropy behavior in Fe3O4 films, Phys. Rev. B, 53 (1996) 91759187.

[15] D.L. Peng, T. Asai, N. Nozawa, T. Hihara, K. Sumiyama, Magnetic properties and magnetoresistance in small iron oxide cluster assemblies, Appl. Phys. Lett., 81 (2002) 45984600 .

[16] G.F. Goya, T.S. Berquó, F.C. Fonseca, M.P. Morales, Static and dynamic magnetic properties of spherical magnetite nanoparticles, J. Appl. Phys., 94 (2003) 3520-3528.

[17] Q.A. Pankhurst, J. Connolly, S.K. Jones, J. Dobson, Applications of magnetic nanoparticles in biomedicine, J. Phys. D: Appl. Phys., 36 (2003) R167.

[18] M. Venkatesan, S. Nawka, S.C. Pillai, J.M.D. Coey, Enhanced magnetoresistance in nanocrystalline magnetite, J. Appl. Phys., 93 (2003) 8023-8025.

[19] G. Reiss, A. Hutten, Magnetic nanoparticles: Applications beyond data storage, Nat. Mater., 4 (2005) 725-726. 
[20] C.A. Kleint, H.C. Semmelhack, M. Lorenz, M.K. Krause, Structural and magnetic properties of epitaxial magnetite thin films prepared by pulsed laser deposition, J. Magn. Magn. Mater., 140-144 (1995) 725-726.

[21] G.Q. Gong, A. Gupta, G. Xiao, W. Qian, V.P. Dravid, Magnetoresistance and magnetic properties of epitaxial magnetite thin films, Phys. Rev. B, 56 (1997) 5096-5099.

[22] S.P. Sena, R.A. Lindley, H.J. Blythe, C. Sauer, M. Al-Kafarji, G.A. Gehring, Investigation of magnetite thin films produced by pulsed laser deposition, J. Magn. Magn. Mater., 176 (1997) 111-126.

[23] X.W. Li, A. Gupta, G. Xiao, G.Q. Gong, Transport and magnetic properties of epitaxial and polycrystalline magnetite thin films, J. Appl. Phys., 83 (1998) 7049-7051.

[24] M.L. Paramês, Z. Viskadourakis, J. Giapintzakis, M.S. Rogalski, O. Conde, Magnetoresistance of magnetite thin films grown by pulsed laser deposition on $\mathrm{GaAs}\left(\begin{array}{lll}1 & 0 & 0\end{array}\right)$ and A12O3(0 00 1), Appl. Surf. Sci., 254 (2007) 1255-1259.

[25] R.N. Goyal, D. Kaur, A.K. Pandey, Substrate dependent structural and magnetic properties of pulsed laser deposited Fe $3 \mathrm{O} 4$ thin films, J. Nanosci. Nanotechnol., 10 (2010) 8018-8025.

[26] S. Kale, S.M. Bhagat, S.E. Lofland, T. Scabarozi, S.B. Ogale, A. Orozco, S.R. Shinde, T. Venkatesan, B. Hannoyer, B. Mercey, W. Prellier, Film thickness and temperature dependence of the magnetic properties of pulsed-laser-deposited Fe3O4 films on different substrates, Phys. Rev. B, 64 (2001) 2054131-2054139.

[27] M.L. Paramês, J. Mariano, Z. Viskadourakis, N. Popovici, M.S. Rogalski, J. Giapintzakis, O. Conde, PLD of Fe3O4 thin films: Influence of background gas on surface morphology and magnetic properties, Appl. Surf. Sci., 252 (2006) 4610-4614.

[28] J.J. Lin, S. Mahmood, T. Zhang, S.M. Hassan, T. White, R.V. Ramanujan, P. Lee, R.S. Rawat, Synthesis of Fe 304 nanostructures by backward plume deposition and influence of ambient gas pressure on their morphology, J. Phys. D: Appl. Phys., 40 (2007) 2548-2554.

[29] S.B. Ogale, K. Ghosh, R.P. Sharma, R.L. Greene, R. Ramesh, T. Venkatesan, Magnetotransport anisotropy effects in epitaxial magnetite (Fe 3O 4) thin films, Phys. Rev. B, 57 (1998) 7823-7828.

[30] J. Tang, K.Y. Wang, W. Zhou, Magnetic properties of nanocrystalline Fe3O4 films, J. Appl. Phys., 89 (2001) 7690-7692.

[31] A. Bollero, M. Ziese, R. Höhne, H.C. Semmelhack, U. Köhler, A. Setzer, P. Esquinazi, Influence of thickness on microstructural and magnetic properties in $\mathrm{Fe} 3 \mathrm{O} 4$ thin films produced by PLD, J. Magn. Magn. Mater., 285 (2005) 279-289.

[32] M. Bohra, N. Venkataramani, S. Prasad, N. Kumar, D.S. Misra, S.C. Sahoo, R. Krishnan, Study of pulsed laser deposited magnetite thin film, J. Magn. Magn. Mater., 310 (2007) 22422244.

[33] R. Prakash, R.J. Choudhary, L.S. Sharath Chandra, N. Lakshmi, D.M. Phase, Electrical and magnetic transport properties of $\mathrm{Fe} 3 \mathrm{O} 4$ thin films on a $\mathrm{GaAs}(100)$ substrate, J. Phys.:Cond. Matter, 19 (2007).

[34] S. Tiwari, R. Prakash, R.J. Choudhary, D.M. Phase, Oriented growth of Fe 304 thin film on crystalline and amorphous substrates by pulsed laser deposition, J. Phys. D: Appl. Phys., 40 (2007) 4943-4947.

[35] M.H. Mahmoud, M.A. Ahmed, Mössbauer study of the pulsed laser deposition of polycrystalline magnetic films, J. Magn. Magn. Mater., 320 (2008) 2647-2649. 
[36] S. Tiwari, R.J. Choudhary, D.M. Phase, Effect of growth temperature on the structural and transport properties of magnetite thin films prepared by pulse laser deposition on single crystal $\mathrm{Si}$ substrate, Thin Solid Films, 517 (2009) 3253-3256.

[37] M. Alexe, M. Ziese, D. Hesse, P. Esquinazi, K. Yamauchi, T. Fukushima, S. Picozzi, U. Gösele, Ferroelectric Switching in Multiferroic Magnetite (Fe3O4) Thin Films, Adv. Mater., 21 (2009) 4452-4455.

[38] R. Master, S. Tiwari, R.J. Choudhary, U.P. Deshpande, T. Shripathi, D.M. Phase, Infrared spectroscopic study of pulsed laser deposited Fe $3 \mathrm{O} 4$ thin film on Si (111) substrate across Verwey transition temperature, J. Appl. Phys., 109 (2011).

[39] A. Hamie, Y. Dumont, E. Popova, A. Fouchet, B. Warot-Fonrose, C. Gatel, E. Chikoidze, J. Scola, B. Berini, N. Keller, Investigation of high quality magnetite thin films grown on SrTiO3(001) substrates by pulsed laser deposition, Thin Solid Films, 525 (2012) 115-120.

[40] J.-G. Yun, Y.-M. Lee, W.-J. Lee, C.-S. Kim, S.-G. Yoon, Selective growth of pure magnetite thin films and/or nanowires grown in situ at a low temperature by pulsed laser deposition, Journal of Materials Chemistry C, 1 (2013) 1977-1982.

[41] M. Walczak, M. Oujja, J.F. Marco, M. Sanz, M. Castillejo, Pulsed laser deposition of TiO2: Diagnostic of the plume and characterization of nanostructured deposits, Appl. Phys. A, 93 (2008) 735-740.

[42] D.L.A. de Faria, S. Venâncio Silva, M.T. de Oliveira, Raman microspectroscopy of some iron oxides and oxyhydroxides, J. Raman Spectroc., 28 (1997) 873-878.

[43] R.E. Vandenberghe, C.A. Barrero, G.M. da Costa, E. Van San, E. De Grave, Mössbauer characterization of iron oxides and (oxy)hydroxides: the present state of the art, Hyperfine Interact., 126 (2000) 247-259.

[44] O.N. Shebanova, P. Lazor, Raman study of magnetite (Fe3O4): laser-induced thermal effects and oxidation, J. Raman Spectroc., 34 (2003) 845-852.

[45] R. Kelly, A. Miotello, Comments on explosive mechanisms of laser sputtering, Appl. Surf. Sci., 96-98 (1996) 205-215.

[46] L.J. Lewis, D. Perez, Laser ablation with short and ultrashort laser pulses: Basic mechanisms from molecular-dynamics simulations, Appl. Surf. Sci., 255 (2009) 5101-5106.

[47] I. Balberg, H.L. Pinch, The optical absorption of iron oxides, J. Magn. Magn. Mater., 7 (1978) 12-15. 